Proyecciones

Vol. 21, $\mathrm{N}^{o}$ 1, pp. 21-50, May 2002.

Universidad Católica del Norte

Antofagasta - Chile

\title{
SOME SPECIAL KLEINIAN GROUPS AND THEIR ORBIFOLDS *
}

\author{
RUBÉN HIDALGO \\ Universidad Técnica Federico Santa María - Chile
}

\begin{abstract}
Let us consider an abstract group with the following presentation

$$
G=\left\langle x_{1}, \ldots, x_{n} ; x_{i}^{k_{i}}=\left(x_{j+1} x_{j}^{-1}\right)^{l_{j}}=1\right\rangle,
$$

where $k_{i}, l_{j} \in\{2, \ldots, \infty\}$. We provide conditions in order to find a faithful, discrete and geometrically finite representation $\Theta: G \rightarrow P S L(2, \mathbf{C})$, that is, to represent $G$ as a group of isometries of the hyperbolic three space $\mathbf{H}^{3}$.
\end{abstract}

*This work was partially supported by projects Fondecyt 1000715, Fondecyt 1010093 and UTFSM 12.01.22. 


\section{Introduction}

The main problem of three orbifolds is their classification. W.P. Thurston [10] observed that geometry and topology are very well related. There are exactly eight geometries [9], the hyperbolic one being the most important. Thurston's conjecture is essentially that all three orbifolds can be obtained by gluing a finite number of geometric orbifolds (that is, obtained by quotient of some of the geometries by a discrete group of isometries). To understand this classification problem, is good to have explicit examples of geometric orbifolds. For it, triangle and Coxeter groups have been of great interest (see CoxeterMoser [1]). Another examples are given by generalized triangle groups $[2],[3],[4],[5]$. In this paper, we consider certain type of generalized Coxeter groups (see next section for definitions). A theorical study of these groups can be found in [11] and [12]. In [6] we have considered a generalized Coxeter group of type

$$
G=\left\langle x_{1}, \ldots, x_{n}: x_{j}^{k}=\left(x_{j+1}^{-1} x_{j}\right)^{l}=1\right\rangle,
$$

and obtained necessary and sufficient conditions to find a faithful, discrete representation of $G$ into $\operatorname{PSL}(2, \mathbf{C})$. We also described the hyperbolic three orbifolds they uniformize. In this note, we consider the more general situation, that is,

$$
G=\left\langle x_{1}, \ldots, x_{n}: x_{j}^{k_{j}}=\left(x_{j+1}^{-1} x_{j}\right)^{l_{j}}=1\right\rangle,
$$

where $k_{j}, l_{j} \in\{2,3, \ldots\} \cup\{\infty\}$. In section 2 we recall the definition on generalized Coxeter groups and we define the ones we are interested. In section 3 we describe a construction of certain hyperbolic polyhedra called $n$-pyramids. The group generated by the reflections on the faces of such a $n$-pyramid is a Coxeter group containing as index two orientation preserving transformations a group of the above type. In section 4 we consider as example the particular case $n=3$ (that is, tetrahedra) of finite volume. We list all possible finite volume hyperbolic tetrahedra with integer quotients of $\pi$ as dihedral angles. In this way, we get faithful discrete and geometrically finite representations of finite volume $\Theta: G \rightarrow P S L(2, \mathbf{C})$, for

$$
G=\left\langle x, y, z: x^{a}=y^{b}=z^{c}=\left(y^{-1} x\right)^{d}=\left(z^{-1} y\right)^{e}=\left(x^{-1} z\right)^{f}=1\right\rangle .
$$


In section 5 , we recall the symmetric case, that is, $k_{j}=k$ and $l_{j}=l$, of [6]: For $n \geq 3$ and $l>\frac{n}{n-2}$, there are faithful discrete and geometrically finite representations $\Theta: G \rightarrow P S L(2, \mathbf{C})$. In this case, the representations extends to a faithful discrete and geometrically finite one $\Theta_{1}: K \rightarrow P S L(2, \mathbf{C})$, where

$$
K=\left\langle x, y: x^{k}=\left(y x^{-1} y^{-1} x\right)^{l}=1\right\rangle
$$

is the group containing $G$ as index $n$ subgroup $\left(x_{1}=x\right.$ and $x_{j+1}=$ $\left.y x_{j} y^{-1}\right)$. In the case $k \neq 2$, we also can extend the representation to a faithful and discrete one $\Theta_{2}: H \rightarrow P S L(2, \mathbf{C})$, where

$$
H=\left\langle x_{1}, \ldots, x_{n}, t: x_{j}^{k}=\left(x_{j+1}^{-1} x_{j}\right)^{l}=\left(t x_{j}\right)^{2}=1\right\rangle .
$$

We must note in this case that the group $H$ contains as index two the group $L=\left\langle x_{1}, \ldots, x_{n}, z: x_{j}^{k}=\left(x_{j+1}^{-1} x_{j}\right)^{l}=\left(y x_{j}\right)^{k}=1\right\rangle$, where $z=t^{2}$. Combining the above two representations, we obtain a faithful discrete one $\Theta_{3}: T \rightarrow P S L(2, \mathbf{C})$, where

$$
T=\left\langle x, y, t: x^{k}=y^{n}=(t x)^{2}=t y^{-1} t^{-1} y=\left(y^{-1} x^{-1} y x\right)^{l}=1\right\rangle .
$$

Selberg's lemma asserts that each finitely generated discrete group of isometries of $\mathbf{H}^{3}$ contains a finite index torsion free normal subgroup. Such a subgroup uniformizes a hyperbolic 3-dimensional manifold. In section 6 we proceed to find such a torsion free normal subgroup of finite index for some of the above embedded groups. In section 7 we describe a second construction for embedding $G$ when $k_{j}=\infty$ and $\sum_{i=1}^{n} l_{j}<(n-2) \pi$.

\section{Generalized Coxeter Groups}

A generalized Coxeter group $G$ is by definition an abstract group with generators $x_{1}, \ldots, x_{n}$, and relations of the form:

(1) $x_{i}^{k_{i}}=1$, where $k_{i} \in\{2,3, \ldots\} \cup\{\infty\}$, for each $i=1, \ldots, n$;

(2) $\left(x_{i}^{-1} x_{j}\right)^{l_{i j}}=1$, where $l_{i j}=l_{j i} \in\{2,3, \ldots\} \cup\{\infty\}$, for each $i \neq$ $j \in\{1,2, \ldots, n\}$. 
If we have $k_{i}=2$, for all $i \in\{1, \ldots, n\}$, then a generalized Coxeter group is in fact a Coxeter group.

As for Coxeter groups, each generalized Coxeter group has associated a generalized Coxeter diagram (the only difference with the definition of Coxeter diagram is that we assign a value $k_{i}$ to each vertex $\left.x_{i}\right)$. For instance, $G=\left\langle x_{1}, x_{2}: x_{1}^{2}=x_{2}^{3}=\left(x_{2}^{-1} x_{1}\right)^{3}=1\right\rangle$ is a generalized Coxeter group (this is the alternating group $\mathcal{A}_{4}$ ). Its generalized Coxeter diagram is formed by an edge labelled with the value 3 , joining two vertices $x_{1}$ and $x_{2}$ labelled by 2 and 3 , respectively, as it is shown in figure 1.

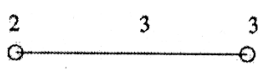

Figure 1

Coxeter groups are natural for the construction of groups generated by reflections on the sides of some polyhedron. Generalized Coxeter groups appear as the index two orientation-preserving subgroup.

A natural way to construct groups of isometries of the hyperbolic three space $\mathbf{H}^{3}$ is by considering a convex hyperbolic polyhedron $Q$ together the reflections on its faces. Necessary and sufficient conditions for the group generated by these reflections to be discrete is to have all internal angles of $Q$ as integral quotients of $\pi$ (tangencies are aloud). The sufficiency part is consequence of the Maskit-Poincare's polyhedron theorem (see page 73 in [7]) and the necessary part is given by the facts that (i) the composition of two reflections along faces meeting at angle $\theta$ will give a rotation along their intersection line on angle $2 \theta$; and (ii) a discrete group only may have finite order rotations.

Let $\widetilde{G}$ be the group generated by the reflections on the sides of the polyhedron $Q$ (satisfying the above property on the internal angles) and $G$ be the index two subgroup of orientation-preserving isometries.

The group $\widetilde{G}$ is a Coxeter group with Coxeter diagram obtained directly from the shape of the polyhedron $Q$. The group $G$ is a generalized Coxeter group generated by the products $x_{i}=\sigma_{0} \sigma_{i}$, where $\sigma_{0}$ is 
the reflection on a fixed face $e_{0}$ of $Q$, and $\sigma_{i}$ runs over the reflections on the other faces of $Q$. The relations are given by

(1) $x_{i}^{k_{i}}=1$, if the faces $e_{0}$ and $e_{i}$ meet at angle $\frac{\pi}{k_{i}}\left(k_{i}=\infty\right.$ if the faces do not intersect in $\mathbf{H}^{3}$ );

(2) $\left(x_{j} x_{i}^{-1}\right)^{l_{i j}}=1$, if the faces $e_{i}$ and $e_{j}$ meet at angle $\frac{\pi}{l_{i j}}\left(l_{i j}=\infty\right.$ if the faces do not intersect in $\mathbf{H}^{3}$ ).

In this note, we consider a certain type of convex hyperbolic polyhedron $Q \subset \mathbf{H}^{3}$, determined by a collection of circles on the Riemann sphere so that the group $G$ has presentation

$$
\left\langle x_{1}, \ldots, x_{n} ; x_{i}^{k_{i}}=\left(x_{j+1} x_{j}^{-1}\right)^{l_{j}}=1\right\rangle,
$$

where $n \geq 3$, and $k_{i}, l_{j} \in\{2, \ldots, \infty\}$.

The generalized Coxeter diagram of $G$ is given as follows (see figure 2 for $n=6)$ :

(i) the vertices $\in$ are the $n$ root of unity (in the complex plane) labelled by $k_{1}, \ldots, k_{n}$, in counterclockwise order, starting at 1 ; and

(ii) the edges of the graph are the edges of a $n$ sided polygon determined by the above vertices, labelled by $l_{1}, \ldots, l_{n}$, in counterclockwise order, starting from the edge that joins 1 with $e^{\frac{2 \pi}{n}}$, and the edges connecting all non-adjacent vertices, all of them labelled with $\infty$.

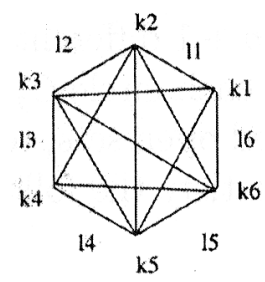

Figure 2 
If we consider $n=2$, then $G$ turns out to be a triangular group (in this case, necessarily, $l_{1}=l_{2}=l$ )

$$
\left\langle x_{1}, x_{2} ; x_{1}^{k_{1}}=x_{2}^{k_{2}}=\left(x_{2} x_{1}^{-1}\right)^{l}=1\right\rangle .
$$

It is a classical fact that triangle groups can be embedded as a group of isometries of the hyperbolic three space. Moreover, the number $\delta=\frac{1}{k_{1}}+\frac{1}{k_{2}}+\frac{1}{l}$ determines the type of group as follows:

(i) if $\delta<1$, then $G$ is hyperbolic;

(ii) if $\delta=1$, then $G$ is Euclidean;

(iii) if $\delta>1$, then $G$ is finite.

In [6] we have shown that, if $k_{i}=k$ and $l_{j}=l$ are integers greater or equal to two, then the generalized Coxeter group $\left\langle x_{1}, \ldots, x_{n} ; x_{i}^{k}=\right.$ $\left.\left(x_{j+1} x_{j}^{-1}\right)^{l}=1\right\rangle$, can be embedded, for $(n, k, l) \neq(3,3,2)$, as a group of isometries of either the hyperbolic three space, the Euclidean three space or the product of the Euclidean line with either the two-sphere or the hyperbolic plane. Moreover, if $(n, k, l)$ is different from $(3,2,2)$, $(3,2,3),(3,3,2)$ and $(4,2,2)$, then we have the hyperbolic situation (see section 5). The case $(3,3,2)$ determines a finite group of order 96 non-isomorphic to a dihedral group,, in particular, cannot be embedded as group of isometries of the hyperbolic three space.

\section{First Construction: n-Pyramids}

Assume that we are given $n+1$ different circles $C_{0}, C_{1}, \ldots, C_{n}(n \geq 3)$ on the complex plane. For each circle $C_{j}$, we denote by $D_{j}^{\text {ext }}$ and $D_{j}^{\text {int }}$ the unbounded and bounded open sets, respectively, determined by it. Assume that these circles satisfy the following properties (see figure 3 for $n=10$ ):

(1) $C_{0}$ and $C_{i}$ intersect transversally for each $i=1, \ldots, n$;

(2) $C_{i}$ and $C_{i \pm 1}$ intersect transversally for each $i=1, \ldots, n(\bmod n)$;

(3) $\left(C_{i} \cup D_{i}^{\text {int }}\right) \cap\left(C_{j} \cup D_{j}^{\text {int }}\right)=\emptyset$, if $j \notin\{i-1, i, i+1\}$; 
(4) The angle between $C_{0}$ and $C_{i}$ determined in $D_{0}^{\text {ext }} \cap D_{i}^{\text {ext }}$ is $\frac{\pi}{k_{i}}$, where $k_{i}$ is an integer greater or equal to two;

(5) The angle between $C_{i}$ and $C_{i+1}$ determined in $D_{i}^{\text {ext }} \cap D_{i+1}^{\text {ext }}$ is $\frac{\pi}{l_{i}}$, where $l_{i}$ is an integer greater or equal to two.

The hyperbolic polyhedron $Q \subset \mathbf{H}^{3}$ bounded by the hyperbolic planes determined by all the above circles is a fundamental polyhedron for this group, which is topologically a truncated $n$-pyramid. We must note that this $n$-pyramid has vertices either in $\mathbf{H}^{3}$, the boundary $\widehat{\mathbf{C}}=$ $\mathbf{C} \cup\{\infty\}$ or beyond infinity (in which case, we say that the pyramid is truncated at that vertex)..

If we denote by $\sigma_{j}$ the reflection on the circle $C_{j}$, then the group $\widetilde{G}$ generated by them is a discrete group of isometries of the hyperbolic space having presentation

$$
\widetilde{G}=\left\langle\sigma_{0}, \ldots, \sigma_{n}: \sigma_{j}^{2}=\left(\sigma_{0} \sigma_{i}\right)^{k_{i}}=\left(\sigma_{i+1} \sigma_{i}\right)^{l_{i}}=1\right\rangle .
$$

The polyhedron $Q$ is a fundamental domain for its action of the hyperbolic space $\mathbf{H}^{3}$. Its index two subgroup $G$ of orientation-preserving isometries has presentation

$$
G=\left\langle x_{1}, \ldots, x_{n}: x_{i}^{k_{i}}=\left(x_{j+1} x_{j}^{-1}\right)^{l_{j}}=1\right\rangle,
$$

where $x_{i}=\sigma_{0} \sigma_{i}$. The hyperbolic polyhedron $Q \cup \sigma_{0}(Q)$ is a fundamental polyhedron for $G$. 


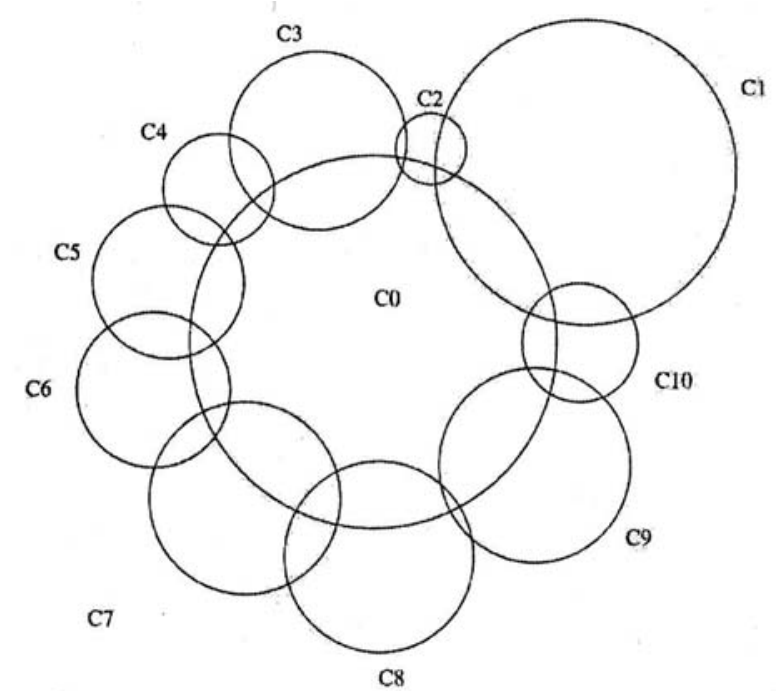

Figure 3

For (i) $n \geq 4$ and (ii) $n=3$ and $\frac{1}{l_{1}}+\frac{1}{l_{2}}+\frac{1}{l_{3}}<1$, the polyhedron $Q \cup \sigma_{0}(Q)$ has at least two ends on the Riemann sphere (vertices beyond the infinite). Each of these ends determines a connected component of the region of discontinuity of $G$. Two of them are permuted by each generator $x_{i}$. The other components have as stabilizer a triangular group. It follows that, under the above assumption, the group $G$ has no invariant component. Moreover, except for the special case $k_{1}=\cdots=k_{n}=2$ (in which case the circle $C_{0}$ is the limit set of $G$ and $\widetilde{G}$ ) the group $G$ has an infinite number of components. The quotient by $G$ of the two components which are permuted has signature $\left(0, n ; l_{1}, \ldots, l_{n}\right)$ and the other quotients are spheres with three branch values. The quotient $\mathbf{H}^{3} / G$ is the orbifold with the three sphere (minus some points) as underlying space and singular locus a planar graph obtained as a $n$-pyramid (the points deleted from the three sphere are some of the vertices of this pyramid). Figure 4 shows the graph $n=6$. 


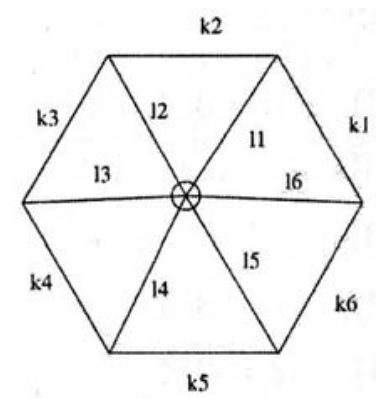

Figure 4

\section{Example: Finite Volume Tetrahedra}

The 3-pyramids we are looking for in the above construction are all possible hyperbolic (truncated) tetrahedra with integer quotients of $\pi$ as angles between its faces. In this section we proceed to recall all possible finite volume of these tetrahedra (that is, without vertices beyond infinite) [8]. In particular, this section describe all finite volume representations of the group

$$
G=\left\langle x, y, z: x^{a}=y^{b}=z^{c}=\left(y^{-1} x\right)^{d}=\left(z^{-1} y\right)^{e}=\left(x^{-1} z\right)^{f}=1\right\rangle .
$$

Let us observe that each possible tetrahedron (not necessarily of finite volume) determines at most 24 presentations as desired for the same group $G$. This is because we can use any of the four faces for the choice of the reflection $\sigma_{0}$ and we have then 6 possibilities for the election of $\sigma_{1}, \sigma_{2}$ and $\sigma_{3}$. We will say that all of them are equivalent.

Theorem 1. Modulo equivalence, all possible finite volume representations of the group $G=\left\langle x, y, z: x^{a}=y^{b}=z^{c}=\left(y^{-1} x\right)^{d}=\right.$ $\left.\left(z^{-1} y\right)^{e}=\left(x^{-1} z\right)^{f}=1\right\rangle$, are given by the following possibilities for $(a, b, c, d, e, f)$ : 
I Non-Compact Cases:

1. $(2,2, c, 3,3,3), \quad c=3,4,5,6$,

2. $(3,3,3,3,3,3)$,

3. $(2,3,3,3,3,3)$,

4. $(2,2, c, 4,4,2), \quad c=3,4$,

5. $(2,2, c, 4,2,4), \quad c=3,4$,

6. $(2, c, 2,4,4,2), \quad c=3,4$,

7. $(2,3,2,3,6,2)$,

8. $(3,2, c, 4,4,2), \quad c=3,4$,

9. $(4,2,4,4,4,2)$,

10. $(3,2,2,2,3,6)$,

11. $(c, 2,2,2,6,3), \quad c=3,4,5,6$,

12. $(c, 2,3,3,6,2), \quad c=3,4,5,6$,

\section{Compact Cases:}

1. $(2,2,3,5,3,2)$,

2. $(2,2, c, 5,2,3), \quad c=4,5$,

3. $(3,5,3,2,2,2)$,

4. $(2, c, 3,3,2, d), \quad c=4,5, d=3,4$

5. $(2,5,3,3,2,5)$

Proof : Let us consider a hyperbolic tetrahedron with faces $F_{1}$, $F_{2}, F_{3}$ and $F_{4}$, so that the angle between the faces $F_{i}$ and $F_{j}$ is $\pi / r_{i, j}$, where $r_{i, j} \in\{2,3, \ldots\}$ (see figure 5 ). If we want this tetrahedron to have finite volume, we also need to have:

(1) $\frac{1}{r_{1,4}}+\frac{1}{r_{3,4}}+\frac{1}{r_{1,3}} \geq 1$ 


$$
\begin{aligned}
& \text { (2) } \frac{1}{r_{1,4}}+\frac{1}{r_{2,4}}+\frac{1}{r_{1,2}} \geq 1 \\
& \text { (3) } \frac{1}{r_{2,4}}+\frac{1}{r_{3,4}}+\frac{1}{r_{2,3}} \geq 1 . \\
& \text { (4) } \frac{1}{r_{1,2}}+\frac{1}{r_{2,3}}+\frac{1}{r_{1,3}} \geq 1 .
\end{aligned}
$$

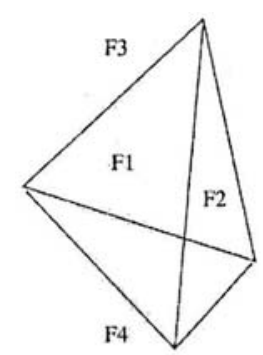

\section{Figure 5}

If we consider the hyperbolic plane $H \subset \mathbf{H}^{3}$ containing the face $F_{4}$, then the faces $F_{1}, F_{2}$ and $F_{3}$ determine a hyperbolic triangle on $H$. Let us denote by $\theta_{i} \in(0, \pi)$ the angle of this triangle formed by the lines $f_{i}$ and $f_{i+1}$, where $f_{i}=F_{i} \cap F_{4}$ for $i=1,2,3$. We have that

$$
\begin{aligned}
\text { (5) } \cos \left(\frac{\pi}{r_{1,2}}\right) & =-\cos \left(\frac{\pi}{r_{1,4}}\right) \cos \left(\frac{\pi}{r_{2,4}}\right)+\cos \left(\theta_{1}\right) \sin \left(\frac{\pi}{r_{1,4}}\right) \sin \left(\frac{\pi}{r_{2,4}}\right) ; \\
\text { (6) } \cos \left(\frac{\pi}{r_{2,3}}\right) & =-\cos \left(\frac{\pi}{r_{2,4}}\right) \cos \left(\frac{\pi}{r_{3,4}}\right)+\cos \left(\theta_{2}\right) \sin \left(\frac{\pi}{r_{2,4}}\right) \sin \left(\frac{\pi}{r_{3,4}}\right) ; \\
\text { (7) } \cos \left(\frac{\pi}{r_{1,3}}\right) & =-\cos \left(\frac{\pi}{r_{1,4}}\right) \cos \left(\frac{\pi}{r_{3,4}}\right)+\cos \left(\theta_{3}\right) \sin \left(\frac{\pi}{r_{1,4}}\right) \sin \left(\frac{\pi}{r_{3,4}}\right) .
\end{aligned}
$$

$$
\text { (8) } \theta_{1}+\theta_{2}+\theta_{3}<\pi \text {. }
$$

In fact, to construct a tetrahedron (of finite volume) in $\mathbf{H}^{3}$ as above, we only need to have the existence of a hyperbolic triangle with angles $\theta_{1}, \theta_{2}$ and $\theta_{3}$ satisfying (5), (6) and (7). 
Non-Compact Case. We assume at least one vertex of the tetrahedron on the boundary of the hyperbolic space (see figure 6). We may assume then:

$$
\text { (4) } \frac{1}{r_{1,2}}+\frac{1}{r_{2,3}}+\frac{1}{r_{1,3}}=1
$$

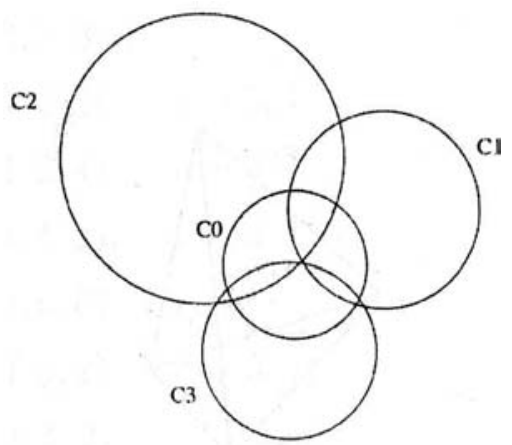

Figure 6

Adding inequalities (1), (2) and (3) gives us

$$
\frac{1}{r_{1,4}}+\frac{1}{r_{2,4}}+\frac{1}{r_{3,4}} \geq 1 \text {. }
$$

In particular, modulo permutation of faces (a topological isometry of the tetrahedron), we obtain the above list.

\section{Compact Case}

If the tetrahedra is compact, then its faces cut the boundary $\widehat{\mathbf{C}}$ as shown in figure 7 . 


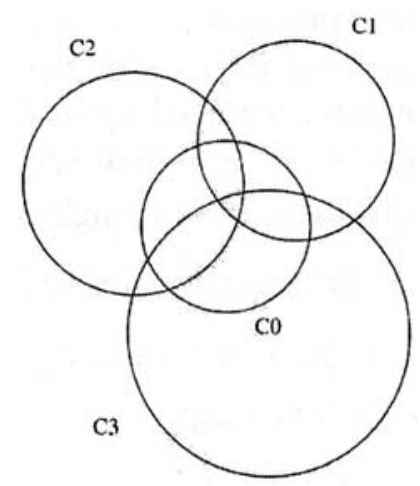

Figure 7

In this case, all possible tetrahedra as desired are given in the above list.

\section{The Symmetric Case}

In this part, we consider the case $n \geq 3$ and $k_{i}=k$ and $l_{j}=l$. Let us assume we have $k, l \in\{2,3, \ldots, \infty\}$, so that $l>\frac{n}{n-2}$.

Under this condition, we are able to construct a regular hyperbolic $n$-gone $F$ in the unit disc model of the hyperbolic plane $\mathbf{H}^{2}$, which is invariant under the rotation $y(z)=e^{\frac{2 \pi}{n}} z$ and with each interior angle $\theta$, where

$$
\cos \left(\frac{\pi}{l}\right)=-\cos \left(\frac{\pi}{k}\right)^{2}+\cos (\theta) \sin \left(\frac{\pi}{k}\right)^{2}
$$

For $k=2$ we have that there is such a regular $n$-gone with $\theta=\frac{\pi}{l}$.

For $k>2$, we have that there is a regular $n$-gone with internal angle $\pi / l$. Denote by $e_{1}, \ldots, e_{n}$ the geodesic lines that determine the boundaries of $F$.

It is easy to check that for $n \geq 4$ the lines $e_{i}$ and $e_{j}$ do not intersect in the closure of the unit disc if $j \neq i-1, i, i+1$. This is consequence of the fact that the sum of internal angles of a hyperbolic triangle is always less than $\pi$. 
Let us consider a circle $C_{r}$, centered at the origin and radius $r \in$ $(0,1]$. The lines $e_{i}$ and the circle $C_{r}$ intersect if and only if $r \in\left[r_{0}, 1\right]$, where $r_{0}$ is given to obtain tangency.

Let us measure the angle of intersection between $e_{i}$ and $C_{r}$ at the common exterior of the discs bounded by each of them. As consequence of the symmetry of $F$, we have that such an angle is the same for all $i=1, \ldots, n$. If we denote by $\theta_{r}$ such angle, we get that $\theta_{r} \in\left[0, \frac{\pi}{2}\right]$, $\theta_{r_{0}}=0, \theta_{1}=\frac{\pi}{2}$ and $\theta_{r}$ is an increasing function on $r \in\left[r_{0}, 1\right]$. It follows that there exists $r_{1} \in\left[r_{0}, 1\right]$ for which $\theta_{r_{1}}=\frac{\pi}{k}$ (if $k=\infty$, then $\left.r_{1}=r_{0}\right)$ and, we obtain the following:

Theorem 2. Let $n \geq 3$ and $k, l \in\{2,3, \ldots, \infty\}$ be so that $l>\frac{n}{n-2}$. Then we can embed the generalized Coxeter group $G=\left\langle x_{1}, \ldots, x_{n}\right.$ : $\left.x_{i}^{k}=\left(x_{i+1}^{-1} x_{i}\right)^{l}=1\right\rangle$ as a group of isometries of $\mathbf{H}^{3}$.

In this symmetric case, we have an extra isometry given by $y$. If we add to $G$ this extra isometry, we get a Kleinian group $K$ with presentation

$$
K=\left\langle x, y: x^{k}=y^{n}=\left(y x^{-1} y^{-1} x\right)^{l}=1\right\rangle,
$$

where $x=x_{1}$, and containing $G$ as a normal subgroup of index $n$. The Riemann surface that $K$ uniformizes has signature $(0,3 ; n, n, l)$ and the hyperbolic orbifold it uniformizes is the three sphere (with some deleted points) and branched locus given by a one-bridge of a link of two circles as shown in figure 8 .

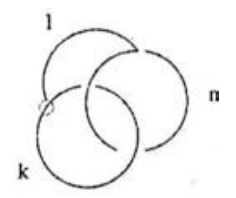

Figure 8 
In the case that $k \neq 2$, if we denote by $\eta$ the reflection on the boundary of the hyperbolic plane $F$, then we have that for $t=\eta \sigma_{0}$ the group $H$ generated by $x_{1}, \ldots, x_{n}$ and $t$ is a group of isometries of $\mathbf{H}^{3}$ with presentation:

$$
H=\left\langle x_{1}, \ldots, x_{n}, t: x_{i}^{k}=\left(x_{i+1}^{-1} x_{i}\right)^{l}=\left(t x_{i}\right)^{2}=1\right\rangle .
$$

In this case, $\mathbf{H}^{3} / H$ is topologically the three sphere (with some deleted points) and branched locus as shown in the following figure for $n=6$ :

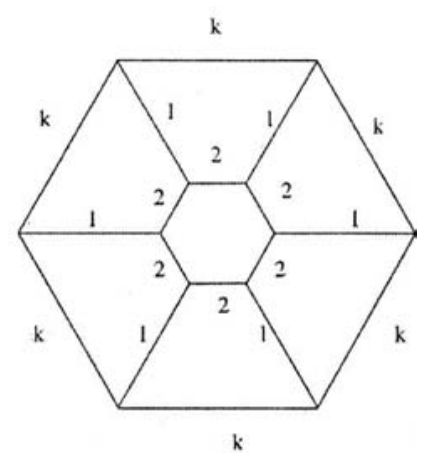

Figure 9

At this point, let us note that $H$ has a subgroup $L$ that also contains $G$ :

$$
L=\left\langle x_{1}, \ldots, x_{n}, z: x_{i}^{k}=\left(x_{i+1}^{-1} x_{i}\right)^{l}=\left(z x_{i}\right)^{k}=1\right\rangle,
$$

$\left(z=t^{2}\right)$ so that the hyperbolic orbifold uniformized by it is the one shown in figure 9 by replacing the values " 2 " by the value " $k$ ".

We also have in the case $k \neq 2$ the group $T$ generated by $H$ and $y$ has presentation (writing $x=x_{1}$ )

$$
T=\left\langle x, y, t: x^{k}=y^{n}=(t x)^{2}=t y^{-1} t^{-1} y=\left(y^{-1} x^{-1} y x\right)^{l}=1\right\rangle
$$

The orbifold $\mathbf{H}^{3} / T$ is topologically the three sphere branched at a graph as shown in figure 9 . 


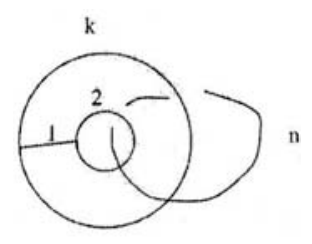

Figure 10

If we consider the group

$$
M=\left\langle x, y, z: x^{k}=y^{n}=(z x)^{k}=z y^{-1} z^{-1} y=\left(y^{-1} x^{-1} y x\right)^{l}=1\right\rangle,
$$

then the orbifold $\mathbf{H}^{3} / M$ is topologically the three sphere branched at a graph as shown in figure 9 by replacing the values " 2 " by the value " $k$ ".

\section{Some examples of Hyperbolic Three-manifolds}

Once we have a generalized Coxeter group $G$ embedded as a group of isometries of the hyperbolic three space $\mathbf{H}^{3}$, Selberg's lemma asserts the existence of a torsion-free normal subgroup $H$ of finite index. The group $H$ uniformizes a hyperbolic three manifold admitting as a group of automorphisms the finite group $G / H$. In the following examples, we proceed to compute explicitly a surjective homomorphism onto some finite group so that its kernel is torsion-free. 


\section{Example 1}

If we consider the symmetric situation with $n=4, l=2$ and $k=3$, we have the group

$$
G=\left\langle x_{1}, x_{2}, x_{3}, x_{4}: x_{1}^{3}=x_{2}^{3}=x_{3}^{3}=x_{4}^{3}=1,\left(x_{2}^{-1} x_{1}\right)^{2}=\left(x_{3}^{-1} x_{2}\right)^{2}=\right.
$$
$\left.\left(x_{4}^{-1} x_{3}\right)^{2}=\left(x_{1}^{-1} x_{4}\right)^{2}=1\right\rangle$.

If we consider the permutations

- $a=(5,4,2)(8,3,6)$,

- $b=(6,3,1)(7,4,5)$

- $c=(2,4,7)(1,8,6)$ and

- $d=(8,1,3)(5,2,7)$,

then we have that $\mathcal{S}_{4}=\langle a, b, c, d\rangle$ and they satisfy the relations

$a^{3}=b^{3}=c^{3}=d^{3}=\left(b^{-1} a\right)^{2}=\left(c^{-1} b\right)^{2}=\left(d^{-1} c\right)^{2}=\left(a^{-1} g\right)^{2}=1$.

Considering the surjective homomorphism $\phi: G \rightarrow \mathcal{S}_{4}$, defined by $\phi\left(x_{1}\right)=a, \phi\left(x_{2}\right)=b, \phi\left(x_{3}\right)=c$ and $\phi\left(x_{4}\right)=d$, we have that its kernel $F$ is a torsion free, finite index normal subgroup of $G$. In particular, $M=\mathbf{H}^{3} / F$ is a non-compact hyperbolic 3-manifold of finite volume admitting the group $\mathcal{S}_{4}$ as group of isometries so that $M / \mathcal{S}_{4}$ is topologically the three dimensional sphere (with five deleted points) branched at a 4-pyramid with branched values 2 and 3 . Moreover, the quotient admits an isometry of order four which can be lifted to $M$. It follows that $M$ admits a group of isometries of order 96 .

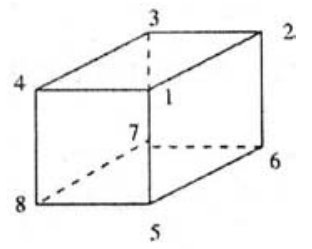

Figure 11 


\section{Example 2 :}

If we consider the symmetric situation with $n=3, l=3$ and $k=4$, we have the group

$G=\left\langle x_{1}, x_{2}, x_{3}: x_{1}^{4}=x_{2}^{4}=x_{3}^{4}=1,\left(x_{2}^{-1} x_{1}\right)^{3}=\left(x_{3}^{-1} x_{2}\right)^{3}=\left(x_{1}^{-1} x_{3}\right)^{3}=1\right\rangle$.

If we consider the permutations (see figure 11):

- $a=(1,2,3,4)(5,6,7,8)$,

- $b=(1,4,8,5)(2,3,7,6)$ and

- $c=(2,1,5,6)(3,4,8,7)$,

then we have that $\mathcal{S}_{4}=\langle a, b, c\rangle$ and they satisfy the relations

$$
a^{4}=b^{4}=c^{4}=\left(b^{-1} a\right)^{3}=\left(c^{-1} b\right)^{3}=\left(a^{-1} c\right)^{2}=1 .
$$

Considering the surjective homomorphism $\phi: G \rightarrow \mathcal{S}_{4}$, defined by $\phi\left(x_{1}\right)=a, \phi\left(x_{2}\right)=b$ and $\phi\left(x_{3}\right)=c$, we have that its kernel $F$ is a torsion free, finite index normal subgroup of $G$. In particular, $M=\mathbf{H}^{3} / F$ is a non-compact hyperbolic 3-manifold of infinite volume admitting the group $\mathcal{S}_{4}$ as group of isometries so that $M / \mathcal{S}_{4}$ is topologically the three dimensional sphere (with four deleted points) branched at a 3-pyramid with branched values 3 and 4 . Moreover, the quotient admits an isometry of order three which can be lifted to $M$. It follows that $M$ admits a group of isometries of order 72

\section{Example 3}

If we consider the symmetric situation with $n=3, l=5$ and $k=5$, we have the group

$G=\left\langle x_{1}, x_{2}, x_{3}: x_{1}^{5}=x_{2}^{5}=x_{3}^{5}=1,\left(x_{2}^{-1} x_{1}\right)^{5}=\left(x_{3}^{-1} x_{2}\right)^{5}=\left(x_{1}^{-1} x_{3}\right)^{5}=1\right\rangle$. 
If we consider the permutations

- $a=(1,2,3,4,5)(8,10,12,14,6)(7,9,11,13,15)(17,18,19,20,16)$,

- $b=(1,5,6,7,8)(17,9,2,4,15)(12,13,20,19,11)(3,14,16,18,10)$ and

- $c=(5,4,14,15,6)(16,7,1,3,13)(9,10,11,19,18)(17,8,2,12,20)$,

then we have that $\mathcal{A}_{5}=\langle a, b, c\rangle$ and they satisfy the relations

$$
a^{5}=b^{5}=c^{5}=\left(b^{-1} a\right)^{5}=\left(c^{-1} b\right)^{3}=\left(a^{-1} c\right)^{2}=1 .
$$

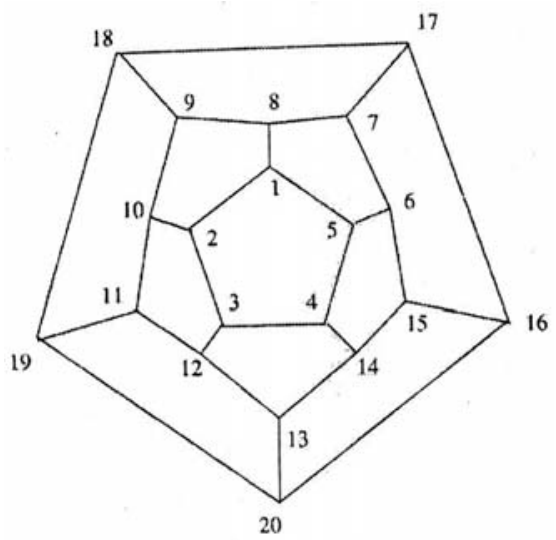

Figure 12

Considering the surjective homomorphism $\phi: G \rightarrow \mathcal{A}_{5}$, defined by $\phi\left(x_{1}\right)=a, \phi\left(x_{2}\right)=b$ and $\phi\left(x_{3}\right)=c$, we have that its kernel $F$ is a torsion free, finite index normal subgroup of $G$. In particular, $M=\mathbf{H}^{3} / F$ is a non-compact hyperbolic 3-manifold of infinite volume admitting the group $\mathcal{A}_{5}$ as group of isometries so that $M / \mathcal{A}_{5}$ is topologically the three dimensional sphere (with four deleted points) branched at a 3 -pyramid with branched values 5. Moreover, the quotient admits an isometry of order three which can be lifted to $M$. It follows that $M$ admits a group of isometries of order 180 . 


\section{Example 4}

If we consider the symmetric situation with $n=5, l=5$ and $k=3$, we have the group

$G=\left\langle x_{1}, x_{2}, x_{3}, x_{4}, x_{5}: x_{1}^{3}=x_{2}^{3}=x_{3}^{3}=x_{4}^{3}=x_{5}^{3}=1,\left(x_{2}^{-1} x_{1}\right)^{5}=\right.$ $\left.\left(x_{3}^{-1} x_{2}\right)^{5}=\left(x_{4}^{-1} x_{3}\right)^{5}=\left(x_{5}^{-1} x_{4}\right)^{5}=\left(x_{1}^{-1} x_{5}\right)^{5}=1\right\rangle$.

If we consider the permutations (see figure 12):

- $a_{1}=(8,5,2)(7,4,10)(6,3,9)(17,14,11)(12,18,15)(19,16,13)$,

- $a_{2}=(10,1,3)(9,5,12)(8,4,11)(18,6,13)(14,19,7)(20,17,15)$,

- $a_{3}=(12,2,4)(11,1,14)(10,5,13)(19,8,15)(6,20,9)(16,18,7)$,

- $a_{4}=(14,3,5)(13,2,6)(12,1,15)(20,10,7)(8,16,11)(17,19,9)$ and

- $a_{5}=(6,4,1)(15,3,8)(14,2,7)(16,12,9)(10,17,13)(18,20,11)$,

then we have that $\mathcal{A}_{5}=\left\langle a_{1}, a_{2}, a_{3}, a_{4}, a_{5}\right\rangle$ and they satisfy the relations

$a_{1}^{3}=a_{2}^{3}=a_{3}^{3}=a_{4}^{3}=a_{5}^{3}=1,\left(a_{2}^{-1} a_{1}\right)^{5}=\left(a_{3}^{-1} a_{2}\right)^{5}=\left(a_{4}^{-1} a_{3}\right)^{5}=$ $\left(a_{5}^{-1} a_{4}\right)^{5}=\left(a_{1}^{-1} a_{5}\right)^{5}=1$.

Considering the surjective homomorphism $\phi: G \rightarrow \mathcal{A}_{5}$, defined by $\phi\left(x_{j}\right)=a_{j}$, for $j=1,2,3,4,5$, we have that its kernel $F$ is a torsion free, finite index normal subgroup of $G$. In particular, $M=\mathbf{H}^{3} / F$ is a non-compact hyperbolic 3-manifold of infinite volume admitting the group $\mathcal{A}_{5}$ as group of isometries so that $M / \mathcal{A}_{5}$ is topologically the three dimensional sphere (with six deleted points) branched at a 5-pyramid with branched values 3 and 5 . Moreover, the quotient admits an isometry of order five which can be lifted to $M$. It follows that $M$ admits a group of isometries of order 300 .

\section{Example 5}

If we consider the situation with $n=3, k=3, l_{1}=l_{2}=3, l_{3}=2$, then we have the group

$G=\left\langle x_{1}, x_{2}, x_{3}: x_{1}^{3}=x_{2}^{3}=x_{3}^{3}=1,\left(x_{2}^{-1} x_{1}\right)^{3}=\left(x_{3}^{-1} x_{2}\right)^{3}=\left(x_{1}^{-1} x_{3}\right)^{2}=1\right\rangle$. 
If we consider the permutations

- $a=(2,3,4)$,

- $b=(4,1,3)$ and

- $c=(4,1,2)$,

then we have that $\mathcal{A}_{4}=\langle a, b, c\rangle$ and they satisfy the relations

$$
a^{3}=b^{3}=c^{3}=\left(b^{-1} a\right)^{3}=\left(c^{-1} b\right)^{3}=\left(a^{-1} c\right)^{2}=1 .
$$

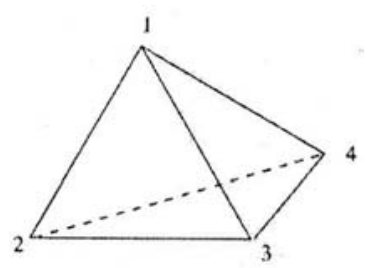

Figure 13

Considering the surjective homomorphism $\phi: G \rightarrow \mathcal{S}_{4}$, defined by $\phi\left(x_{1}\right)=a, \phi\left(x_{2}\right)=b$ and $\phi\left(x_{3}\right)=c$, we have that its kernel $F$ is a torsion free, finite index normal subgroup of $G$. In particular, $M=\mathbf{H}^{3} / F$ is a non-compact hyperbolic 3-manifold of finite volume admitting the group $\mathcal{A}_{4}$ as group of isometries so that $M / \mathcal{A}_{4}$ is topologically the three dimensional sphere (with two deleted points) branched at a 3pyramid with branched values 3 and 2 . Moreover, the quotient admits an isometry of order two which can be lifted to $M$. It follows that $M$ admits a group of isometries of order 24

\section{Example 6}

If we consider the situation with $n=3, k=5, l_{1}=l_{2}=3, l_{3}=5$, then we have the group

$$
G=\left\langle x_{1}, x_{2}, x_{3}: x_{1}^{5}=x_{2}^{5}=x_{3}^{5}=1,\left(x_{2}^{-1} x_{1}\right)^{3}=\left(x_{3}^{-1} x_{2}\right)^{3}=\left(x_{1}^{-1} x_{3}\right)^{5}=1\right\rangle \text {. }
$$


If we consider the permutations (see figure 12):

- $a=(1,2,3,4,5)(8,10,12,14,6)(7,9,11,3,15)(17,18,19,20,16)$,

- $b=(8,7,6,5,1)(15,4,2,9,17)(11,19,20,13,12)(10,18,16,14,3)$ and

- $c=(5,4,14,15,6)(16,7,1,3,13)(9,10,11,19,18)(17,8,2,12,20)$,

then we have that $\mathcal{A}_{5}=\langle a, b, c\rangle$ and they satisfy the relations

$$
a^{5}=b^{5}=c^{5}=\left(b^{-1} a\right)^{3}=\left(c^{-1} b\right)^{3}=\left(a^{-1} c\right)^{5}=1 .
$$

Considering the surjective homomorphism $\phi: G \rightarrow \mathcal{A}_{5}$, defined by $\phi\left(x_{1}\right)=a, \phi\left(x_{2}\right)=b$ and $\phi\left(x_{3}\right)=c$, we have that its kernel $F$ is a torsion free, finite index normal subgroup of $G$. In particular, $M=\mathbf{H}^{3} / F$ is a non-compact hyperbolic 3-manifold of infinite volume admitting the group $\mathcal{A}_{5}$ as group of isometries so that $M / \mathcal{A}_{5}$ is topologically the three dimensional sphere (with four deleted points) branched at a 3-pyramid with branched values 3 and 5 . Moreover, the quotient admits an isometry of order two which can be lifted to $M$. It follows that $M$ admits a group of isometries of order 120 .

\section{Example 7}

Let us consider $l>\frac{n}{n-2}$ and the group

$$
G=\left\langle x_{1}, \ldots, x_{n}: x_{j}^{k}=\left(x_{j+1}^{-1} x_{j}\right)^{l}=1\right\rangle .
$$

Theorem 4 asserts that $G$ can be embedded as group of isometries of $\mathbf{H}^{3}$. In this particular case, the commutator subgroup $[G, G]$ turns out to be a torsion free normal subgroup of index $k l^{n}$, and

$$
G /[G, G] \cong \mathbf{Z} / k \mathbf{Z} \times \mathbf{Z} / l \mathbf{Z} \times \cdots \times \mathbf{Z} / l \mathbf{Z}
$$

In particular, $[G, G]$ uniformizes a hyperbolic three manifold $M$ with a group of automorphisms containing the above finite Abelian group. Also it admits an extra automorphism of order $n$, so it admits a group of order at least $n k l^{n}$ as group of automorphisms. 


\section{Example 8: The compact tetrahedra.}

\section{1}

Let us consider $n=3, l_{1}=2, l_{2}=3, l_{3}=3, k_{1}=3, k_{2}=5$ and $k_{3}=2$. In this case, the group

$G=\left\langle x_{1}, x_{2}, x_{3}: x_{1}^{3}=x_{2}^{5}=x_{3}^{2}=\left(x_{2}^{-1} x_{1}\right)^{2}=\left(x_{3}^{-1} x_{2}\right)^{3}=\left(x_{1}^{-1} x_{3}\right)^{3}=1\right\rangle$,

is a co-compact group of isometries of the hyperbolic space $\mathbf{H}^{3}$. If we set $H$ to be the normalizer of $\left(x_{1} x_{2} x_{3}\right)^{2}$ in $G$, then we get that $H$ is a torsion free normal subgroup of index 60 (this can be obtained using the program GAP). In particular, $M=\mathbf{H}^{3} / H$ is a compact hyperbolic three manifold with a group of isometries $K$ isomorphic to $\mathcal{A}_{5}$, so that $M / K=\mathbf{H}^{3} / G$.

\section{2}

Let us consider $n=3, l_{1}=2, l_{2}=3, l_{3}=4, k_{1}=3, k_{2}=4$ and $k_{3}=2$. In this case, the group

$G=\left\langle x_{1}, x_{2}, x_{3}: x_{1}^{3}=x_{2}^{4}=x_{3}^{2}=\left(x_{2}^{-1} x_{1}\right)^{2}=\left(x_{3}^{-1} x_{2}\right)^{3}=\left(x_{1}^{-1} x_{3}\right)^{4}=1\right\rangle$,

is a co-compact group of isometries of the hyperbolic space $\mathbf{H}^{3}$. If we set $H$ to be the normalizer of $\left(x_{1} x_{2} x_{3}\right)^{2}$ in $G$, then we get that $H$ is a torsion free normal subgroup of index 96 (this can be obtained using the program GAP). In particular, $M=\mathbf{H}^{3} / H$ is a compact hyperbolic three manifold with a group of isometries $K$ of order 96 , so that $M / K=\mathbf{H}^{3} / G$.

\section{3}

Let us consider $n=3, l_{1}=2, l_{2}=3, l_{3}=5, k_{1}=2, k_{2}=5$ and $k_{3}=2$. In this case, the group

$G=\left\langle x_{1}, x_{2}, x_{3}: x_{1}^{2}=x_{2}^{5}=x_{3}^{2}=\left(x_{2}^{-1} x_{1}\right)^{2}=\left(x_{3}^{-1} x_{2}\right)^{3}=\left(x_{1}^{-1} x_{3}\right)^{5}=1\right\rangle$,

is a co-compact group of isometries of the hyperbolic space $\mathbf{H}^{3}$. The hyperbolic volume of $\mathbf{H}^{3} / G$ is

$$
\operatorname{Vol}\left(\mathbf{H}^{3} / G\right)=0.186651 .
$$


If we set $H$ to be the normalizer of $\left(x_{1} x_{2} x_{3}\right)^{3}$ in $G$, then we get that $H$ is a torsion free normal subgroup of index 60 (this can be obtained using the program GAP). In particular, $M=\mathbf{H}^{3} / H$ is a compact hyperbolic three manifold with a group of isometries $K$ isomorphic to $\mathcal{A}_{5}$, so that $M / K=\mathbf{H}^{3} / G$ and

$$
\operatorname{Vol}(M)=11.1991
$$

\section{4}

Let us consider $n=3, l_{1}=2, l_{2}=3, l_{3}=5, k_{1}=3, k_{2}=5$ and $k_{3}=2$. In this case, the group

$G=\left\langle x_{1}, x_{2}, x_{3}: x_{1}^{3}=x_{2}^{5}=x_{3}^{2}=\left(x_{2}^{-1} x_{1}\right)^{2}=\left(x_{3}^{-1} x_{2}\right)^{3}=\left(x_{1}^{-1} x_{3}\right)^{5}=1\right\rangle$,

is a co-compact group of isometries of the hyperbolic space $\mathbf{H}^{3}$. If we set $H$ to be the normalizer of $\left(x_{1} x_{2} x_{3}\right)^{2}$ in $G$, then we get that $H$ is a torsion free normal subgroup of index 60 (this can be obtained using the program GAP). In particular, $M=\mathbf{H}^{3} / H$ is a compact hyperbolic three manifold with a group of isometries $K$ isomorphic to $\mathcal{A}_{5}$, so that $M / K=\mathbf{H}^{3} / G$.

\section{5}

Let us consider $n=3, l_{1}=l_{2}=l_{3}=2, k_{1}=k_{3}=3$ and $k_{2}=5$. In $\mathrm{t}$ his case, the group

$G=\left\langle x_{1}, x_{2}, x_{3}: x_{1}^{3}=x_{2}^{5}=x_{3}^{3}=1,\left(x_{2}^{-1} x_{1}\right)^{2}=\left(x_{3}^{-1} x_{2}\right)^{2}=\left(x_{1}^{-1} x_{3}\right)^{2}=1\right\rangle$,

is a co-compact group of isometries of the hyperbolic space $\mathbf{H}^{3}$. We have that $\mathbf{H}^{3} / G$ is the three sphere branched at a tetrahedron with three edges (with a common vertex) of order 2 and the others three of orders 3,3 and 5 . If we set $H$ to be the normalizer of $\left(x_{1} x_{2} x_{3}\right)^{3}$ in $G$, then we get that $H$ is a torsion free normal subgroup of index 660 (this can be obtained using the program GAP). In particular, $M=\mathbf{H}^{3} / H$ is a compact hyperbolic three manifold with a group of isometries $K$ of order 660 , so that $M / K=\mathbf{H}^{3} / G$. 


\section{6}

Let us consider $n=3, l_{1}=l_{2}=2, l_{3}=3, k_{1}=2, k_{2}=3$ and $k_{3}=5$. In this case, the group

$G=\left\langle x_{1}, x_{2}, x_{3}: x_{1}^{2}=x_{2}^{3}=x_{3}^{5}=\left(x_{2}^{-1} x_{1}\right)^{2}=\left(x_{3}^{-1} x_{2}\right)^{2}=\left(x_{1}^{-1} x_{3}\right)^{3}=1\right\rangle$,

is a co-compact group of isometries of the hyperbolic space $\mathbf{H}^{3}$. If we set $H$ to be the normalizer of $\left(x_{1} x_{2} x_{3}\right)^{4}$ in $G$, then we get that $H$ is a torsion free normal subgroup of index 360 (this can be obtained using the program GAP). In particular, $M=\mathbf{H}^{3} / H$ is a compact hyperbolic three manifold with a group of isometries $K$ of order 360 , so that $M / K=\mathbf{H}^{3} / G$.

\section{7}

Let us consider $n=3, l_{1}=2, l_{2}=3, l_{3}=3, k_{1}=3, k_{2}=4$ and $k_{3}=2$. In this case, the group

$G=\left\langle x_{1}, x_{2}, x_{3}: x_{1}^{3}=x_{2}^{4}=x_{3}^{2}=\left(x_{2}^{-1} x_{1}\right)^{2}=\left(x_{3}^{-1} x_{2}\right)^{3}=\left(x_{1}^{-1} x_{3}\right)^{3}=1\right\rangle$,

is a co-compact group of isometries of the hyperbolic space $\mathbf{H}^{3}$.

If we set $H$ to be the normalizer of $\left(x_{1} x_{2} x_{3}\right)^{4}$ in $G$, then we get that $H$ is a torsion free normal subgroup of index 2448 (this can be obtained using the program GAP). In particular, $M=\mathbf{H}^{3} / H$ is a compact hyperbolic three manifold with a group of isometries $K$ of order 2448, so that $M / K=\mathbf{H}^{3} / G$.

\section{8}

Let us consider $n=3, l_{1}=2, l_{2}=3, l_{3}=5, k_{1}=2, k_{2}=54$ and $k_{3}=2$. In this case, the group

$G=\left\langle x_{1}, x_{2}, x_{3}: x_{1}^{2}=x_{2}^{4}=x_{3}^{2}=\left(x_{2}^{-1} x_{1}\right)^{2}=\left(x_{3}^{-1} x_{2}\right)^{3}=\left(x_{1}^{-1} x_{3}\right)^{5}=1\right\rangle$,

is a co-compact group of isometries of the hyperbolic space $\mathbf{H}^{3}$ and

$$
\operatorname{Vol}\left(\mathbf{H}^{3} / G\right)=0.0717702 .
$$

If we set $H$ to be the normalizer of $\left(x_{1} x_{2} x_{3}\right)^{6}$ in $G$, then we get that $H$ is a torsion free normal subgroup of index 6840 (this can be 
obtained using the program GAP). In particular, $M=\mathbf{H}^{3} / H$ is a compact hyperbolic three manifold with a group of isometries $K$ of order 6840 , so that $M / K=\mathbf{H}^{3} / G$ and

$$
\operatorname{Vol}(M)=490.908
$$

\section{Second Construction: Infinite Volume}

Let us start with a configuration of circles $C_{0}, C_{1}, \ldots, C_{n}, D_{0}, D_{1}, \ldots$, $D_{n}$, where $n \geq 3$, so that:

(1) $C_{j} \cap D_{i}=\emptyset$;

(2) $C_{j}$ is orthogonal to $C_{0}$, for $j=1, \ldots, n$;

(3) $D_{j}$ is orthogonal to $D_{0}$, for $j=1, \ldots, n$;

(4) $C_{j} \cap C_{i}=\emptyset$, for $i, j \in\{1, \ldots, n\}$ and $j \notin\{i-1, i, 1+1\}$;

(5) $D_{j} \cap D_{i}=\emptyset$, for $i, j \in\{1, \ldots, n\}$ and $j \notin\{i-1, i, 1+1\}$;

(6) $C_{i}$ and $C_{i+1}$ intersect at an angle of the form $\frac{\pi}{l_{i}}$, where $l_{i} \in$ $\{2, \ldots\} \cup\{\infty\}$;

(7) $D_{i}$ and $D_{i+1}$ intersect with angle $\frac{\pi}{l_{i}}$.

(8) There is a Jordan curve $\gamma$, contained in some of the two polygons determined by the configuration of circles $C_{1}, \ldots, C_{n}$, so that the configuration of circles $D_{0}, \ldots, D_{n}$ is contained in the topological disc determined by $\gamma$ that does not contains the other configuration of circles $C_{j}$. 


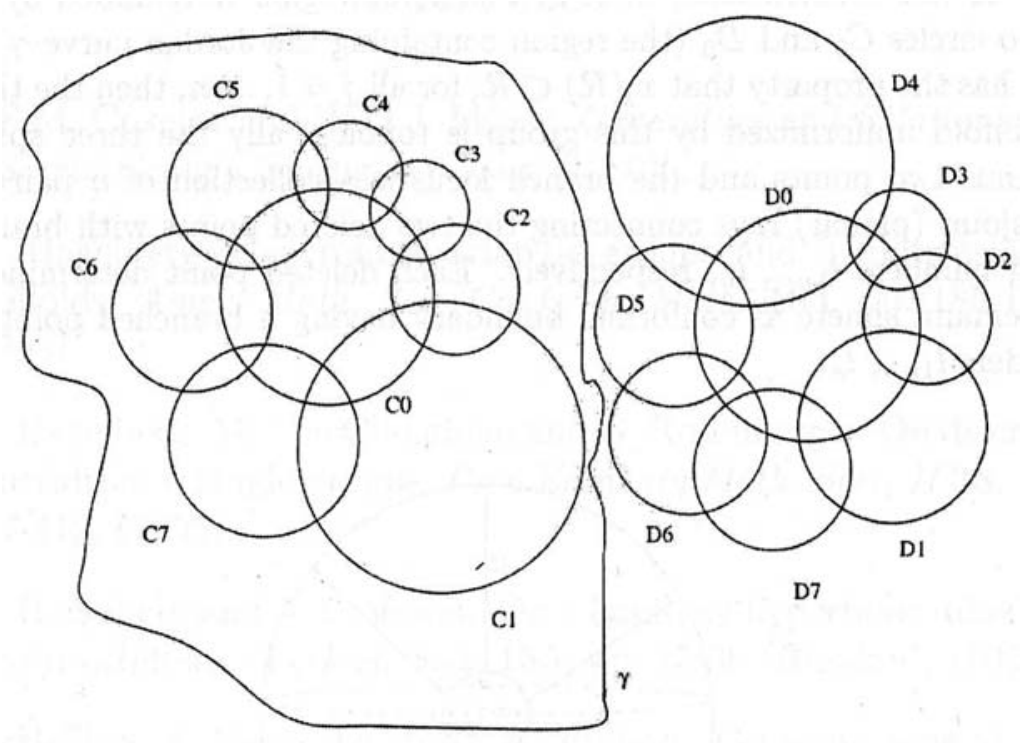

Figure 14

For each $j \in\{1,2, \ldots, n\}$, we choose a loxodromic transformation $x_{j}$ so that

(1) $x_{j}\left(C_{j}\right)=D_{j}$;

(2) $x_{j}\left(C_{j} \cap C_{j+1}\right)=D_{j} \cap D_{j+1}$.

As an application of the Klein-Maskit's combination theorems [7], we have that the group $G$ generated by $x_{1}, \ldots, x_{n}$, is a Kleinian group with fundamental polyhedron domain given by the hyperbolic polyhedron bounded by the geodesic planes determined by the circles $C_{1}, \ldots$, $C_{n}, D_{1}, \ldots, D_{n}$. Moreover, the group $G$ has presentation

$$
G=\left\langle x_{1}, \ldots, x_{n}:\left(x_{j+1}^{-1} x_{j}\right)^{l_{j}}=1\right\rangle .
$$


It follows that this is the type of groups we are considering with the property that $k_{j}=\infty$, for all $j=1, \ldots, n$.

In this construction, there is a common region $\mathcal{R}$ bounded by the two circles $C_{0}$ and $D_{0}$ (the region containing the Jordan curve $\gamma$ ). If $x_{j}$ has the property that $x_{j}(\mathcal{R}) \subset \mathcal{R}$, for all $j=1, \ldots, n$, then the three orbifold uniformized by this group is topologically the three sphere minus two points and the branch locus is a collection of $n$ pairwise disjoint (planar) rays connecting the two deleted points with branching numbers $l_{1}, \ldots, l_{n}$, respectively. Each deleted point determines a Riemann sphere as conformal boundary having $n$ branched points of orders $l_{1}, \ldots, l_{n}$.

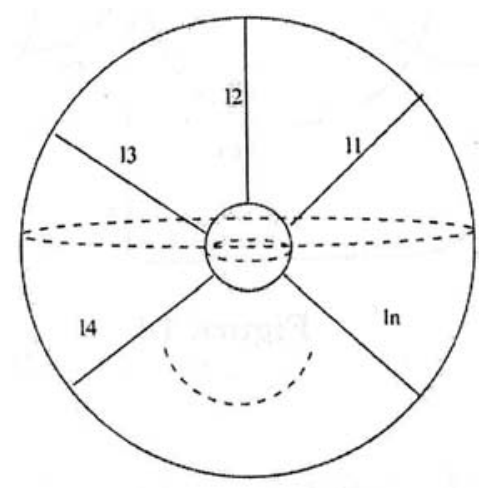

Figure 15

\section{Ending remark}

The general case, that is $n \geq 4$ and non-symmetric, is more complicated. Already in the case $n=4$ it is not easy to write nice inequalities on the values $k_{i}$ and $l_{j}$ to ensure the non-intersecting of the circles $C_{i}$ and $C_{j}$ for $j \notin\{i-1, i, i+1\}$. 


\section{References}

[1] H.S.M. Coxeter and W.O.J. Moser. Generators and Relations for Discrete Groups. Springer-Verlag, (1980).

[2] M. Hagelberg. Generalized triangle groups and 3-dimensional orbifolds. Amer. Math. Soc. Contemp. Math. 184, pp. 185-192, (1995).

[3] M. Hagelberg, M. MacClaughlan and G. Rosenberger. On discrete generalized triangle groups. Proc Edinburg Math. Soc., II 38, pp. 397-412, (1995).

[4] M. Hagelberg and A.Y. Vesnin. On a family of hyperbolic dihedral $\theta(p / q)$-orbifolds. Vychisl. Sist. 155, pp. 15-36 (Russian), (1996).

[5] H. Helling, J. Mennicke and E.B. Vinberg. On some general triangle groups and 3-dimensional orbifolds. Trans. Mosc. Math. Soc., pp. 1-21, (1995).

[6] M. Hagelberg and R. Hidalgo. Generalized Coxeter groups and their orbifolds. Revista Matemática Iberoamericana 13 (1997), pp. 543-566, (1997).

[7] B. Maskit. Kleinian groups. Springer-Verlag, Berlin and New York, (1972).

[8] J.G. Ractliffe. Foundations of Hyperbolic Manifolds. Graduate Texts in Math., Springer-Verlag, (1994).

[9] W. Scott. The geometries of three manifolds. Bull. London Math. Soc. 15, pp. 407-487, (1983).

[10] W.P. Thurston. The geometry and topology of 3-manifolds. Lecture Notes, Princeton Univ.., (1980).

[11] S.V. Tsaranov. On a generalization of Coxeter groups. Alg. Groups Geom. 6, 281-318 (1989). 
[12] . Finite generalize Coxeter groups. Alg. Groups Geom. 6, pp. 421452, (1989).

Received : May 2001.

Rubén Hidalgo

Departamento de Matemática

Universidad Técnica Federico Santa María

Casilla 110-V

Valparaíso

Chile

e-mail : rhidalgo@mat.utfsm.cl 